Archives de sciences sociales des religions

127 | juillet - septembre 2004

Max Weber, la religion et la construction du social

\title{
Le concept d'affinité élective chez Max Weber
}

\section{Michael Löwy}

\section{OpenEdition}

Journals

Édition électronique

URL : http://journals.openedition.org/assr/1055

DOI : 10.4000/assr.1055

ISSN : $1777-5825$

Éditeur

Éditions de l'EHESS

Édition imprimée

Date de publication : 1 juillet 2004

Pagination : 93-103

ISBN : 2-222-96751-1

ISSN : 0335-5985

Référence électronique

Michael Löwy, «Le concept d'affinité élective chez Max Weber », Archives de sciences sociales des religions [En ligne], 127 | juillet - septembre 2004, mis en ligne le 25 juin 2007, consulté le 10 décembre 2020. URL : http://journals.openedition.org/assr/1055 ; DOI : https://doi.org/10.4000/assr.1055 


\section{LE CONCEPT D'AFFINITÉ ÉLECTIVE CHEZ MAX WEBER}

Rares sont les chercheurs en sociologie des religions qui n'ont pas, en commentant les écrits de Weber, et en particulier L'Éthique Protestante, constaté l'utilisation du terme "affinité élective ». Mais, étrangement, ce terme n'a pas suscité d'études, discussions ou débats ; on ne trouve même pas un repérage systématique des passages où l'expression est présente. Et encore moins une analyse un peu plus systématique de sa signification méthodologique. Ces notes sont une première tentative, encore très partielle et inachevée, de combler cette lacune (1).

Le terme affinité élective (Wahlverwandtschaft) a une longue histoire qui est bien antérieure aux écrits de Weber. Essayons de reconstituer brièvement cet itinéraire complexe, pour pouvoir capter toute la richesse de significations qu'il a accumulée au cours de son étrange périple culturel, qui va de l'alchimie à la littérature romantique, et de celle-ci aux sciences sociales.

C'est dans l'alchimie médiévale qu'on commence à utiliser le terme affinité pour expliquer l'attraction et la fusion des corps. Selon Albert le Grand, si le souffre s'unit aux métaux, c'est à cause de l'affinité qu'il possède avec ces corps : propter affinitatem naturae metalla adurit. On retrouve cette thématique chez les alchimistes au cours des siècles suivants. Par exemple, dans son livre Elementa Chimiae (1724), Hermanus Boerhave explique que particulae solventes et solutae se affinitate sue naturae colligunt in corpora homogenea. L'affinité est une force en vertu de laquelle deux substances diverses "se recherchent, s'unissent et se retrouvent", dans une sorte de mariage, de noce chimique, procédant plutôt de l'amour que de la haine, magis ex amore quam ex odio (2).

(1) Il existe un essai de Richard Herbert Howe, «Max Weber's Elective Affinities. Sociology within the bounds of pure reason ", American Journal of sociology, no 84, 1978, qui contient des informations intéressantes sur les origines du terme, mais la définition qu'il propose - l'affinité élective comme « une idée au sens kantien »-n'est pas très pertinente. En outre, il semble vouloir la réduire à une «affinité élective entre mots », en fonction de «l'intersection de leurs significations », ce qui en limite considérablement la portée. (Ibid. pp. $366 ; 382$ ).

(2) Hermannus Boerhave, Elementa Chimiae, Lugduni Batavorum, 1732, Part II, « De Menstruis ». 
Le terme attractionis electivae apparaît pour la première fois chez le chimiste suédois Torbern Olof Bergman. Son livre, De attractionibus electivis (Uppsala, 1775), sera traduit en français sous le titre Traité des affinités chimiques ou attractions électives (1788). Dans la traduction allemande - Francfort, Verlag Tabor, 1782-1790 - le terme "attraction élective" sera rendu par Wahlverwandtschaft, affinité élective.

C'est probablement dans cette version allemande de T.O. Bergman que Goethe a puisé le titre de son roman Die Wahlverwandtschaften (1809), où il est question d'un ouvrage de chimie étudié par un des personnages "environ il y a une dizaine d'années". Le terme devient ici une métaphore pour désigner le mouvement passionnel par lequel un homme et une femme sont attirés l'un par l'autre - quitte à se séparer de leurs partenaires antérieurs - à partir de l'affinité intime entre leurs âmes. Cette transposition, par Goethe, du concept chimique sur le terrain social de la spiritualité et de l'amour était d'autant plus aisée que, chez plusieurs alchimistes, comme Boerhave, le terme était déjà fortement chargé de métaphores sentimentales et érotiques. Pour Goethe, il y a affinité élective quand deux êtres ou éléments "se cherchent l'un l'autre, s'attirent, se saisissent l'un l'autre et ensuite ressurgissent de cette union intime dans une forme renouvelée et imprévue" (3). La ressemblance avec la formule de Boerhave - deux éléments qui "se recherchent, s'unissent et se retrouvent" - est frappante, et il n'est pas exclu que Goethe ait connu aussi l'œuvre de l'alchimiste néerlandais et qu'il s'en soit inspiré.

Avec le roman de Goethe le terme a gagné droit de cité dans la culture allemande, comme désignation d'un type de lien particulier entre les âmes. C'est donc en Allemagne qu'il connaîtra sa troisième métamorphose : la transmutation, par l'œuvre de ce grand alchimiste de la science sociale qui s'appelle Max Weber, en concept sociologique. Il garde de l'acception ancienne les connotations de choix réciproque, attirance et combinaison, mais la dimension de la nouveauté semble disparaître (4).

Le concept occupe une place importante dans l'œuvre “classique" de Weber, L'Éthique protestante et l'esprit du capitalisme, précisément pour analyser le rapport complexe et subtil entre ces deux formes. Il s'agit pour Weber de dépasser l'approche traditionnelle en termes de causalité, et de contourner ainsi le débat sur la primauté du "matériel" ou du "spirituel" : " face à l'extraordinaire enchevêtrement d'influences réciproques entre les substrats matériels, les formes d'organisation sociale et politique et la teneur intellectuelle des époques culturelles de la Réforme, la seule manière de procéder ne peut consister qu'à examiner d'abord si et sur quels points on peut déceler des 'affinités électives' (Wahlverwandtschaften) entre certaines formes de la foi religieuse et l'éthique de la profession-vocation. Par là se trouvent du même coup précisés, autant qu'il est possible, le mode et la direction générale selon lesquels, par suite de telles affinités

(3) Johann Wolfgang GoETHE, Die Wahlverwandtschaften, Gutersloh, C. Bertelsmann Verlag, 1948 , p. 41.

(4) Pour un développement plus détaillé sur les origines du concept, je renvoie à mon livre Rédemption et Utopie. Messianisme juif et utopies libertaires en Europe centrale. Une étude d'affinité élective, Paris, PUF, 1988. 
électives, le mouvement religieux a agi sur le développement de la culture matérielle $\gg(5)$.

Il est intéressant de noter que la première fois le terme apparaît encore entre guillemets, comme si Weber voulait s'excuser de cette référence incongrue à une métaphore romanesque dans une analyse scientifique. Mais dans la suite du paragraphe les guillemets tombent: l'expression a gagné droit de cité dans l'univers conceptuel du sociologue.

Ce qu'il essaye de montrer c'est, tout d'abord, l'existence d'éléments convergents et analogues entre une éthique religieuse et un comportement économique : l'ascétisme puritain et l'épargne du capital, l'éthique protestante du travail et la discipline bourgeoise du travail, la valorisation calviniste du métier vertueux et l'ethos de l'entreprise bourgeoise rationnelle, la conception ascétique de l'usage utilitaire des richesses et l'accumulation productive du capital, l'exigence puritaine de vie méthodique et systématique, et la poursuite rationnelle du profit capitaliste.

C'est à partir de ces analogies profondes, ces «parentés intimes » qu'en Hollande, en Angleterre et aux Etats-Unis, du XVII ${ }^{e}$ au XIX ${ }^{\mathrm{e}}$ siècle, un rapport d'affinité élective entre l'éthique protestante et l'esprit du capitalisme va se développer, grâce auquel la conception puritaine de l'existence favorisera la tendance à une vie bourgeoise économiquement rationnelle - et vice-versa.

Il n'est pas étonnant que l'expression «affinité élective »n'ait pas été comprise par la réception anglo-saxonne positiviste de Max Weber. Un exemple presque caricatural est la traduction américaine de L'Éthique protestante par Talcott Parsons (en 1930): Wahlverwandtschaften a été rendu, tantôt par certain correlations, tantôt par those relationships (6). Tandis que le concept wébérien renvoie à une relation interne riche et significative entre deux configurations, la "traduction" de Parsons le remplace par une banale corrélation extérieure et vide de sens.

Nous ne pouvons pas examiner dans le cadre de cet article dans quelle mesure le terme, ou du moins l'idée de l'affinité élective apparaissent chez d'autres sociologues allemands de cette époque : Tönnies, Simmel, Troeltsch, Mannheim. Par exemple, chez ce dernier on trouve des analyses qui sont proches de la Wahlverwandtschaft wébérienne, même si le terme lui-même n'est pas utilisé : "Dans la confluence (zusammenfliessen) de deux orientations de pensée, la tâche de la sociologie de la connaissance est de trouver les moments dans les deux courants, qui encore avant la synthèse révélaient une affinité interne (innere Verwandtschaft) et qui ont donc rendu possible l'unification" (7).

Weber n'utilise le concept que trois fois dans L'Éthique protestante, mais il apparaît aussi dans d'autres écrits, dans la plupart des cas dans le domaine de la

(5) Max Weber, L'Éthique protestante et l'esprit du capitalisme, (traduit par Jean-Pierre Grossein), Paris, Gallimard, 2004, p. 91. Cf. Max WeBer, Gesammelte Aufsätze zur Religionssoziologie, I, Tübingen, JCB Mohr, 1922, p. 153.

(6) Max Weber, The Protestant Ethic and the Spirit of Capitalism, Londres, Unwin University Books, 1957, pp. 91-92 (trad. Talcott Parsons).

(7) K. Mannheim, « Das Konservative Denken » (1927), Wissensoziologie, Berlin, Luchterhand. 1964, p. 458. L’idée apparaît aussi chez Troeltsch : cf. Jean SÉGuY, Christianisme et Société. Introduction à la sociologie de Ernst Troeltsch, Paris, Cerf, 1980, pp. 247-251. 
sociologie des religions. Sans prétention à l'exhaustivité, on peut signaler dix modalités distinctes de l'affinité élective dans ses écrits. Trois sont internes à un champ déterminé, les autres «traversent» différents champs sociaux :

\section{1) Interne au champ religieux}

Il s'agit du rapport de Wahlverwandtschaft entre des formes religieuses distinctes : par exemple, entre le ritualisme et la grâce sacramentale, ou entre la prophétie de mission et « une conception déterminée du divin : celle d'un dieu créateur, transcendant, personnel, fulminant, pardonnant, aimant, exigeant, châtiant» - en opposition à la divinité impersonnelle et contemplative de la prophétie exemplaire (8).

\section{2) Interne au champ économique}

Entre l' «esprit» du capitalisme et les formes d'organisation économiques capitalistes. Cela peut paraître redondant, mais Weber insiste sur le fait que, de la même façon qu'une organisation syndicale n'est pas toujours animée par un esprit syndicaliste, ni un empire colonial par l'esprit de l'impérialisme, une économie capitaliste n'est pas nécessairement mue par «l'esprit du capitalisme » (9). Selon les cas l' « esprit » se trouve, à un degré ou autre, en adéquation et, éventuellement, en « rapport d'affinité élective » avec la « forme» (10).

\section{3) Interne au champ culturel}

Il s'agit d'un exemple curieux : Weber oppose la formation (Bildung) patrimoniale, qui conduit, en se rationalisant, vers la bureaucratie moderne - spécialisation, professionnalisation - à la Bildung féodale, « avec ses traits ludiques et son affinité élective avec l'activité artistique (Künstlertum). (11)» Weber pense sans doute à l'éducation de l'aristocratie, mais les traits communs avec la pratique de l'art ne sont pas explicités.

\section{4) Entre les formes structurelles de l'action communautaire et les formes concrètes de l'économie}

Cette modalité, suggérée dans le chapitre sur l'essence de l'économie d'Économie et Société (12) reste très générale : Weber n'en donne pas des exemples concrets ; il affirme qu'il va s'y référer souvent dans la continuation du livre, mais ce n'est pas tellement le cas. À moins de considérer les affinités électives entre structures de l'action religieuse et de l'action économique comme une des formes possibles de cette configuration générale.

(8) Max Weber, Sociologie des religions (SR), Textes réunis, traduits et présentés par Jean-Pierre Grossein, Paris, Gallimard, 1996, p. 356.

(9) $S R$ pp. 134-135.

(10) Max Weber, Protestantische Ethik und der Geist des Kapitalismus. II. Kritiken und Antikritiken, (PE II) Munnich-Hambourg, 1968, p. 171.

(11) Wirtschaft und Gesellschaft, Tübingen, JCB Mohr, 1922, p. 752.

(12) Max Weber, Économie et Société, Paris, Plon, 1971, p. 354. 


\section{5) Entre éthique religieuse et ethos économique}

C'est le cas des passages les plus connus, ceux de L'Éthique Protestante, mais on trouve des références semblables dans d'autres écrits de Weber. Par exemple, dans Économie et Société, où il est question d'un rapport plus général entre rationalité économique et éthique religieuse rigoriste :

« Nous laisserons de côté pour l'instant le genre de relation causale qui règne entre une éthique religieuse rationnelle et un type particulier de rationalisme commercial, là où une telle relation existe. Nous voulons seulement établir qu'il existe une affinité élective entre le rationalisme économique et certains types de religiosité éthique rigoriste que nous caractériserons ultérieurement avec plus de précision. Cette affinité ne s'observe qu'occasionnellement en dehors de l'Occident, c'est-à-dire en dehors du terroir du rationalisme économique ; elle se fait de plus en plus nette dès que l'on se rapproche des porteurs classiques du rationalisme économique » (13).

Comme le texte de L'Éthique protestante, celui-ci distingue aussi, explicitement, entre l'affinité élective et la relation causale.

\section{6) Entre formes religieuses et formes politiques}

Les références de ce type sont rares. Un des exemples les plus intéressants est l'affinité élective entre le fonctionnement des sectes et la démocratie: «L'affinité élective intime avec la structure de la démocratie apparaît déjà dans les principes mêmes de structuration propres à la secte ", comme par exemple, la gestion directe par la communauté ou la définition des agents ecclésiastiques comme « serviteurs » du groupe (14).

\section{7) Entre structures économiques et formes politiques}

Le seul exemple que nous avons trouvé est de type "négatif», mais il est hautement significatif. Dans son essai sur la situation de la démocratie bourgeoise en Russie (1906), Weber s'insurge contre un lieu commun de la pensée libérale/bourgeoisie : le lien naturel entre capitalisme et démocratie. Selon Weber, « il est hautement ridicule d'attribuer au grand capitalisme d'aujourd'hui, qui est actuellement importé en Russie, et établi aux États-Unis (...) une affinité élective avec la 'démocratie' ou même avec la 'liberté' (dans tous les sens possibles de ce mot), tandis que la question devrait être : comme ces choses sont-elles mêmes «possibles », à long terme, sous sa domination?»(15) Nous n'avons pas trouvé chez Weber d'exemples "positifs » d'affinité élective entre économie et politique - un enjeu «classique » du matérialisme historique, mais souvent (pas toujours !) abordé sous un angle « déterministe».

\section{8) Entre classes sociales et ordres religieux}

C'est un thème discuté dans Économie et Société: «L'affinité élective générale entre les puissances bourgeoises et les puissances religieuses, qui est typique d'un certain stade de développement de ces deux puissances, peut aller jusqu'à leur

(13) Max Weber, Économie et Société, p. 502.

(14) Max Weber, Économie et Société in SR, p. 324.

(15) Max Weber, "Zur Lage der bürgerlichen Demokratie in Russland», Archiv für Sozialwissenschaft und Sozialpolitik, Band 22, 1906, Beiheft, p. 353. 
alliance formelle contre les pouvoirs féodaux, comme ce fut assez souvent le cas en Orient, ainsi qu'en Italie au temps de la querelle des Investitures ». Il ne s'agit pas simplement d'un pacte entre groupes sociaux en fonction d'intérêts communs, mais d'un ethos pacifique commun, en opposition à la noblesse militaire (16).

\section{9) Entre visions du monde et intérêts de classes sociales}

Le problème est formulé dans les termes les plus généraux dans les Essais sur la Théorie de la Science: les Weltanschauungen ont une autonomie propre, et ne peuvent pas être déduits de telle ou telle position de classe ; cependant, l'adhésion de l'individu à une vision du monde dépend, dans une large mesure, de la Wahlverwandtschaft de celle-ci avec ses intérêts de classe (17).

C'est à ce type de relation que se réfère la définition proposée par Jean Séguy : «L'“affinité élective' selon Weber exprime une double 'chance' sociale : celle qui a à voir un rapport constant s'établir entre une forme d'idéologie (ici de religion) et les intérêts d'une classe économique ou de statut; d'autre part, la 'chance' non moins significative qui existe pour que la flexibilité des structures et de l'agir social empêche ce rapport d'être nécessaire » (18). La formulation est pertinente, mais ne concerne qu'une des modalités de la Wahlverwandtschaft chez Weber (19).

\section{0) Entre les styles de vie d'une classe sociale et certains styles de vie religieux}

Ce cas de figure ne doit pas être confondu avec le précédent : il ne s'agit pas ici d'intérêts seulement, mais de quelque chose de plus vaste : " l'affinité élective du style de vie requis par la religion avec le style de vie socialement conditionné des corps (Stände) et des classes ». L'exemple sur lequel Weber revient toujours est celui, déjà suggéré dans L'Éthique Protestante, et réaffirmé dans les réponses et anti-critiques: le puritanisme ascétique et la moyenne bourgeoisie. D'une façon plus générale, Weber pense que, lors des crises eschatologiques, les adhésions aux mouvements religieux traversent les différentes classes sociales (sont « verticales »), tandis qu'avec leur routinisation, ces adhésions deviennent « horizontales », c'est-àdire obéissent aux lignes de fractures des classes sociales - un fait qui justifie, dans une certaine mesure, "l'interprétation matérialiste historique » (20).

Cette classification n'est pas inutile, mais elle doit être relativisée.

La frontière entre ces différentes modalités n'est pas étanche, et l'on trouve souvent des recoupements: l'ethos économique, les styles de vie des classes sociales et leurs intérêts matériels sont loin d'être toujours des aspects distincts de

(16) Économie et Société, p. 245

(17) Max WeBer, Gesammelte Aufsätze zur Wissenschaftslehre, Tübingen, JCB Mohr, 1922, p. 153.

(18) Jean SÉGuY, Christianisme et Société, p. 251.

(19) Le même vaut pour la définition, plus imprécise, proposée par Reinhard Bendix dans sa biographie de Weber : «Weber a souvent utilisé le terme 'affinité élective' pour exprimer l'aspect double des idées, i.e. qu'elles sont créées ou choisies par l'individu ('électives') et qu'elles correspondent avec ses intérêts matériels ('affinité'). (Reinhard BENDIX, Max Weber, an intellectual portrait, New York, Doubleday, 1962, p. 54).

(20) SR, p 279 et PE II, p. 181. 
la réalité sociale. Par exemple, quand Weber écrit, dans L'Éthique Protestante, que «le calvinisme apparaît dans une plus grande affinité élective avec le sens fermement légaliste et activiste de l'entrepreneur capitaliste-bourgeois " (21) il est difficile de savoir s'il s'agit des intérêts du bourgeois, du style de vie de sa classe, ou de l' " esprit du capitalisme ».

Je n'ai pas essayé de vérifier la pertinence ou non, la vraisemblance ou pas, l'exactitude ou l'erreur de ces différentes hypothèses. Mon objectif ici est plus limité et plus modeste : rendre compte de la logique de la démarche de Weber, de la façon dont il utilise le concept et le fait «travailler ». Cela dit, j'ai laissé de côté certains usages du terme Wahlverwandtschaft par Weber qui me semblent d'un faible intérêt heuristique: lorsqu'il mentionne l'affinité élective entre «les idéaux » des collaborateurs d'une revue de sciences sociales (trop vague) ou entre l'esprit autoritaire d'un parti catholique conservateur (le Zentrum) et les formes d'état autoritaires en Allemagne (trop évident) (22).

Dans aucun de ces exemples Weber ne définit le concept d' « affinité élective ». Il semble le considérer comme allant de soi, suffisamment familier à un public cultivé allemand connaissant par cœur les écrits de Goethe. Mais il donne, ici ou là, quelques précieuses indications sur son «fonctionnement»: quand les deux éléments - par exemple, un système social et un "esprit » culturel - sont liés par « un 'degré d'adéquation' particulièrement élevé » et entrent en rapport d'affinité élective, ils s'adaptent ou s'assimilent réciproquement (aneindander anzugleichen trachten), jusqu'à ce que, « finalement, un développement d'une intime et inébranlable unité s'installe »(23). Ou alors, le degré de Wahlverwandtschaft entre une action communautaire et une forme économique dépend du type de lien actif qui s'établit entre les deux : «si, et avec quelle intensité, elles favorisent réciproquement leur existence, ou, au contraire, l'entravent ou l'excluent; sont réciproquement 'adéquats' ou 'inadéquats'. Nous parlerons souvent de tels rapports d'adéquation. (24)»

Comme nous avons vu dans différentes citations, il ne s'agit pas, aux yeux de Weber, d'une relation causale. Si dans tel ou tel passage de L'Éthique Protestante Weber semble tantôt privilégier le rôle efficace des causes économiques, tantôt celui des motivations religieuses, l'orientation méthodologique principale du livre n'affirme ni la priorité du facteur économique ("matériel") ni celle du religieux ("spirituel") mais plutôt leur congruence et leur attraction réciproque. Le concept d'affinité élective permet donc à Weber d'éviter les explications strictement " matérialistes » ou " spiritualistes », qui ne lui semblent pas aptes à rendre compte de la complexité historique des rapports entre les comportements religieux et économiques. Comme l'a bien saisi Jean Séguy, l'utilisation du concept d'affinité

(21) Max Weber, L'Éthique Protestante... p. 173. Celle-ci est la troisième et dernière apparition du terme dans ce livre.

(22) Respectivement dans Max WeBER, Wissenschaftslehre, p. 159 et Gesammelte Politische Schriften, München, Drei Masken Verlag 1921, p. 185.

(23) Max WeBer, PE II, p. 171.

(24) Max WeBER, Wirtschaft und Gesellschaft, p. 183. La traduction française de ce passage (Économie et Société, p. 354) est très approximative. Par exemple, Wahlverwandtschaft est traduit par " affinité » tout court... 
élective est inséparable de la démarche pluraliste de Weber, de son refus de toute monocausalité et de tout déterminisme unilatéral (25).

Puisqu'il n'y a pas de définition, pourrait-on, comme semble le croire JeanPierre Grossein, le traducteur - par ailleurs fort compétent - des Essais de sociologie religieuse, considérer l'affinité élective simplement comme un synonyme de «parenté intérieure »? À son avis, « les commentateurs se sont beaucoup fatigués - inutilement - sur cette notion de Wahlverwandtschaft à haut rendement littéraire. Sont équivalents chez Weber: 'affinité élective', 'adéquation', 'parenté intérieure' » (26). On se demande, dans ce cas, pourquoi Weber a insisté en utilisant cette notion «littéraire », plutôt que de se limiter à ces autres termes, censés être " équivalents ». On trouve un raisonnement analogue chez Raymond Aron, qui définit l'affinité élective comme une forme d' "adéquation significative », d' " affinité spirituelle» ou de «conformité intellectuelle et existentielle» entre une attitude religieuse et un comportement économique (27).

En fait, Weber utilise plusieurs concepts, autour de, ou à propos de l'affinité élective : adéquation, parenté intérieure, affinité de sens, congruence. Ils aident à la compréhension du phénomène, mais ne sont pas, au sens strict, des synonymes ou des équivalents : ils ne peuvent pas remplacer le concept de Wahlverwandtschaft. Ce sont des termes plus faibles, moins chargés de signification et, surtout, dépourvus de la dimension active. Prenons comme exemple le terme «parenté interne » : il désigne bien une Verwandtschaft, une affinité intime, une adéquation entre deux formes, mais pas de rapport actif entre elles. L'affinité élective, par contre, contient l'élément de la sélection, du choix actif, de l'attraction réciproque. Dans le langage de Goethe, auquel Weber fait implicitement référence en utilisant cette expression, les deux formes culturelles « se cherchent l'une l'autre, s'attirent, se saisissent l'une l'autre ». Pour Weber, qui est avant tout un sociologue de l'action, cette différence entre la simple «affinité » et l'affinité élective, entre une analogie formelle et un rapport actif ne pouvait pas passer inaperçue (28).

Je propose la définition suivante, à partir de l'usage wébérien du terme : l'affinité élective est le processus par lequel deux formes culturelles - religieuses, intellectuelles, politiques ou économiques - entrent, à partir de certaines analogies significatives, parentés intimes ou affinités de sens, dans un rapport d'attraction et influence réciproques, choix mutuel, convergence active et renforcement mutuel.

Il faut prendre en considération que l'affinité élective comporte plusieurs niveaux ou degrés :

(25) Jean SÉGuY, Conflit et utopie, ou réformer l'Église. Parcours wébérien en douze essais, Paris, Cerf, 1999, pp. 76-98.

(26) Jean-Pierre Grossein, «Présentation », SR p. 59. $540 ; 542$

(27) Raymond Aron, Les étapes de la pensée sociologique, Paris, Gallimard, 1967, pp. 537 ; 539 ;

(28) Il me semble que Jean-Pierre Grossein n'est pas loin de partager cette conclusion quand il écrit, citant Weber, au sujet de l'affinité élective : «l'adéquation au plan du sens, telle que l'établit une analyse au plan du sens, ne garantit pas l'existence effective d'un tel rapport 'dans chaque cas particulier, ni même dans la majorité ou la moyenne des cas'. » (Jean-Pierre Grossern, «Présentation », SR, p. 60). La citation de Weber se trouve dans $S R$, p.135, à propos du rapport entre « esprit » et « forme » du capitalisme. 
1. L’affinité tout court, la parenté spirituelle, la congruence, l'adéquation interne.

Il est important de souligner que l'affinité est une analogie encore statique, qui crée la possibilité mais non la nécessité d'une convergence active, d'une attraction élective. La transformation de cette puissance en acte, la dynamisation de l'analogie dépend de conditions historiques et sociales concrètes.

Pour donner un exemple évident: Weber constate, entre le confucianisme et le rationalisme puritain « une certaine parenté (Verwandtschaft)» (29). Cela ne suffit pas pour créer entre les deux un rapport effectif de convergence.

2. L'élection, l'attirance réciproque, le choix actif mutuel de deux configurations socioculturelles, conduisant à certaines formes d'interaction, de stimulation réciproque et de convergence. À ce degré, les analogies et correspondances commencent à devenir dynamiques, mais les deux structures demeurent séparées.

3. L'articulation, combinaison ou union entre les partenaires, pouvant résulter dans une sorte de "symbiose culturelle", où les deux figures restent distinctes mais sont organiquement associées ; c'est-à-dire, quand, pour citer Weber, «un développement d'une intime et inébranlable unité s'installe ».

C'est quelque part entre ces deux derniers niveaux que se situe la Wahlverwandtschaft entre éthique protestante et esprit du capitalisme dont parle Weber.

Bien entendu, l'affinité élective dépend du degré d'«adéquation » ou de « parenté » entre les deux formes ; mais elle dépend aussi d'autres facteurs : elle est favorisée ou défavorisée par certaines conditions historiques. En d'autres termes, il faut une certaine constellation - pour utiliser un concept que Mannheim a transplanté avec succès de l'astrologie à la sociologie de la connaissance - de facteurs historiques, sociaux et culturels pour que se déploie un processus d'attractio electiva, de sélection réciproque, renforcement mutuel, et même, dans certains cas, de "symbiose" de deux figures spirituelles. Cette problématique est implicitement présente chez Weber, mais rarement développée.

Il est étonnant que dans l'abondante - immense même - bibliographie autour de la thèse de Weber - la Wahlverwandtschaft entre calvinisme et capitalisme - on trouve si peu d'analyses sur le concept même d'affinité élective. Consultant une bibliographie multilingue sur le débat suscité par le livre de Weber, préparée en 1978 par Johannes Winckelmann, on ne trouve même pas un seul titre d'article ou essai sur ce sujet (30).

Un des rares auteurs qui s'est intéressé à ce thème est le sociologue anglogermanique Werner Stark. Sa démarche est fort ambitieuse : il s'agit d'esquisser les principales lignes d'une théorie des affinités électives, qui serait, dans le domaine des rapports entre les idées et la réalité socio-historique, ou entre « infrastructure » et «superstructure », une alternative - ou du moins un correctif - aux conceptions directement causales ou fonctionnalistes. Le principal objectif de cette théorie présente, selon Stark, non seulement chez Max Weber, mais aussi chez d'autres

(29) Religionssoziologie, I, p. 450.

(30) Cf. Max Weber, Die protestantische Ethik II. Kritiken und Antikritiken, Herausgegeben von Johannes Winckelmann, Gütersloher Verlagshaus, 1978, pp. 395-429, « Bibliographie zur Kontroversialliteratur $»$. 
auteurs comme Max Scheler, Karl Mannheim, Alfred Weber et Alfred von Martin serait la compréhension du lien entre les idées et les classes sociales.

L'affinité élective consiste, selon Stark, «dans une graduelle convergence entre superstructure et infrastructure », comme "deux pôles qui s'attirent réciproquement et entrent, une fois qu'il se sont mutuellement trouvés, dans un attachement durable ». Il s'agit d'un processus d' "adaptation réciproque » dans lequel les deux pôles sont également actifs (31).

Les remarques de cet auteur sont intéressantes, mais curieusement il a très peu à dire sur Weber, dont il ne cite que deux ou trois passages. Son interprétation de la théorie de l'affinité élective se situe avant tout sur le terrain de la sociologie de la connaissance, et elle est plus proche de Max Scheler - selon lequel les sociétés ou les mouvements sociaux ne créent pas leurs conceptions ou leurs philosophies, mais choisissent dans un stock d'idées déjà existantes et indépendantes (dans un sens néo-platonicien) - que de Max Weber.

Il me semble que le concept d'affinité élective peut s'appliquer à beaucoup de domaines. Il permet de comprendre - au sens fort de Verstehen - un certain type de conjonction entre phénomènes apparemment disparates, au sein du même champ culturel (religion, politique, économie) ou entre sphères sociales distinctes : religion et économie, mystique et politique, etc. Il rend compte de processus d'interaction qui ne relèvent ni de la causalité directe, ni du rapport "expressif" entre forme et contenu (par exemple, une forme religieuse étant l'"expression" d'un contenu politique ou social), ni de la «fonction » d'une partie dans un tout social.

Il ne faut pas confondre l'affinité élective, telle qu'on la définit ici, avec la simple parenté idéologique inhérente aux diverses variantes d'un même courant social ou culturel : par exemple, entre libéralisme économique et libéralisme politique, entre socialisme et égalitarisme, entre romantisme littéraire et romantisme social. L'élection, le choix réciproque impliquent une distance préalable, un écart culturel qui doit être comblé, une discontinuité idéologique.

Sans vouloir se substituer aux autres paradigmes analytiques, explicatifs ou compréhensifs, l'affinité élective peut constituer un angle d'approche nouveau, jusqu'ici peu exploré, dans le champ de la sociologie de la culture.

\author{
Michael LÖWY \\ Centre d'Études Interdisciplinaires \\ des Faits Religieux - CNRS
}

(31) Werner Stark, Die Wissensoziologie. Ein Betrag zum tieferen Verständnis des Geistslebens, Stuttgart, Ferdinand Encke Verlag, 1960, pp. 215-222. 


\section{Résumé}

Le terme " affinité élective " (Wahlverwandtschaft) a une longue histoire, largement antérieure aux écrits de Max Weber. Il s'agit d'un itinéraire complexe qui va de l'alchimie à la littérature romantique (Goethe), et de celle-ci aux sciences sociales. Weber n'utilise le concept que trois fois dans L'Éthique protestante, mais il apparaît aussi dans d'autres écrits, dans la plupart des cas dans le domaine de la sociologie des religions. Weber ne le définit pas, mais on pourrait proposer la définition suivante, à partir de l'usage wébérien du terme : l'affinité élective est le processus par lequel deux formes culturelles - religieuses, intellectuelles, politiques ou économiques - entrent, à partir de certaines analogies significatives, parentés intimes ou affinités de sens, dans un rapport d'attraction et influence réciproques, choix mutuel, convergence active et renforcement mutuel. Sans se substituer aux autres paradigmes analytiques, explicatifs ou compréhensifs, l'affinité élective peut constituer un angle d'approche nouveau, jusqu'ici peu exploré, dans le champ de la sociologie de la culture.

\section{Abstract}

The notion of elective affinity (Wahlverwandtschaft) has a long history which is much older that Weber's writings. It went through a complex path that leads from alchemy to romantic literature (Goethe), and from there to the social sciences. Weber uses it only three times in The Protestant Ethic, but it appears also in several of his other writings, mainly concerning sociology of religion. Weber does not define it, but one could propose the following definition, based on the weberian use of the notion: elective affinity is a process through which two cultural forms - religious, intellectual, political or economical - who have certain analogies, intimate kinships or meaning affinities, enter in a relationship of reciprocal attraction and influence, mutual selection, active convergence and mutual reinforcement. Without substituting other analytical, explanatory or comprehensive paradigms, elective affinity may offer a new approach, until now hardly explored, in the field of sociology of culture.

Resumen

La expresion " afinidad electiva" (Wahlverwandtschaft) tiene una larga historia que es bien anterior a los escritos de Max Weber. Se trata de un percurso complejo que va de la alquimia à la literatura romantica (Goethe), y de esta à las ciencias sociales. Weber solo utilisa el concepto três veces en La Etica protestante, pero el aparaece tambien en otros escritos, generalmente en el campo de la sociologia de las religiones. Weber no lo define, pero poderiamos proponer la definicion seguiente, à partir del uso weberiano de la expresion : la afinidad electiva es el proceso por el cual dos formas culturales - religiosas, intelectuales, politicas o economicas - entran, partiendo de ciertas analogias significativas, o afinidades de sentido, en una relacion de atraccion y influencia reciprocas, seleccion mutua, convergencia activa y refuerzo mutuo. Sin sustituirse à otros paradigmas analiticos, explicativos o compreensivos, la afinidad electiva puede constituir una nueva perspectiva, hasta ahora poco explotada, en el campo de la sociologia de la cultura. 
